\title{
A constant conflict
}

\author{
Narrowing down the value of the Hubble constant has been problematic - probably a manifestation of \\ the dark-energy mystery, writes Barbara Ryden.
}

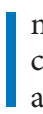
$\mathrm{n}$ the early twentieth century, a class of celestial objects known as spiral nebulae attracted attention because of a remarkable trend in their spectral-line wavelengths. From a list of 41 spiral nebulae compiled by Arthur Eddington in 1923, there were 36 with redshifts and five with blueshifts. If the wavelength shifts were interpreted as Doppler shifts, the mean radial velocity of the spiral nebulae would be $540 \mathrm{~km} \mathrm{~s}^{-1}$. As Eddington wrote ${ }^{1}$, "The great preponderance of positive (receding) velocities is very striking." Although the distances from the Earth to spiral nebulae were initially unknown, in 1924 Edwin Hubble calculated ${ }^{2}$ the distances to the spiral nebulae M31 (the Andromeda galaxy, pictured) and M33 as "about 285,000 parsecs". (One parsec, abbreviated $\mathrm{pc}$, is equal to about $3.1 \times 10^{16} \mathrm{~m}$.) These distance measurements convinced astronomers that spiral nebulae are large, distant galaxies comparable in size to the Milky Way; the rival hypothesis, that spiral nebulae were tiny, nearby satellites of the Milky Way, was abandoned.

By 1929, Hubble had found the distances to 24 spiral galaxies. Plotting the velocity $v$ versus the distance $d$ for these galaxies, he found they could be fitted with a linear relation of the form $v=H_{0} d$, consistent with a homogeneous and isotropic expansion of the Universe ${ }^{3}$. The expansion parameter $H_{0}$, later named the Hubble constant, was calculated by Hubble himself to be $H_{0} \approx 500 \mathrm{~km} \mathrm{~s}^{-1} \mathrm{Mpc}^{-1}$. To determine the distance to galaxies, Hubble used the standard-candle method, based on the inverse square law of flux giving an estimate of the distance to a light source - the 'standard candle' - the luminosity of which is assumed to be known. Hubble's standard candles were actually more luminous than he thought; thus, he was overestimating $H_{0}$.
By 1958, Allan Sandage had corrected some of Hubble's erroneous assumptions, finding a value for the Hubble constant of $H_{0} \approx 75 \mathrm{~km} \mathrm{~s}^{-1} \mathrm{Mpc}^{-1}$, corresponding to a time scale $H_{0}^{-1}$ of about $13 \times 10^{9}$ years ${ }^{4}$. (The value of $H_{0}^{-1}$ provides a ballpark figure for the age of the Universe.) In 1974, Sandage and his collaborator Gustav Tammann worked on an improved determination of the value of $H_{0}$ and arrived at

$50 \mathrm{~km} \mathrm{~s}^{-1} \mathrm{Mpc}^{-1}$ with an error of $10 \%$. At the same time, however, Gérard de Vaucouleurs was also attempting to determine the value of the Hubble constant. Studies by de Vaucouleurs and his collaborators generally led to 